\title{
Approximate decoding for network coded
}

13 inter-dependent data

\author{
${ }^{15}$ Q1 Minhae Kwon ${ }^{\mathrm{a}}$, Hyunggon Park ${ }^{\mathrm{a}}$, Nikolaos Thomos ${ }^{\mathrm{b}}$, Pascal Frossard ${ }^{\mathrm{c}}$ \\ 17 Q2 a Department of Electronics Engineering, Ewha Womans University, Seoul, South Korea \\ ${ }^{\mathrm{b}}$ Computer Science and Electronic Engineering Department, University of Essex, Colchester, United Kingdom \\ Q3 ' Signal Processing Laboratory (LTS4), Ecole Polytechnique Fédérale de Lausanne (EPFL), Lausanne, Switzerland
}

\section{A R T I C L E I N F O}

\section{Article history:}

Received 17 December 2014

Received in revised form

12 July 2015

Accepted 14 September 2015

Keywords:

Network coding

Approximate decoding

Inter-dependence source

Ad hoc networks

33

Loss-tolerant data

\begin{abstract}
A B S T R A C T
In this paper, we consider decoding of loss tolerant data encoded by network coding and transmitted over error-prone networks. Intermediate network nodes typically perform the random linear network coding in a Galois field and a Gaussian elimination is used for decoding process in the terminal nodes. In such settings, conventional decoding approaches can unfortunately not reconstruct any encoded data unless they receive at least as many coded packets as the original number of packets. In this paper, we rather propose to exploit the incomplete data at a receiver without major modifications to the conventional decoding architecture. We study the problem of approximate decoding for inter-dependent sources where the difference between source vectors is characterized by a unimodal distribution. We propose a mode-based algorithm for approximate decoding, where the mode of the source data distribution is used to reconstruct source data. We further improve the mode-based approximate decoding algorithm by using additional short information that is referred to as position similarity information (PSI). We analytically study the impact of PSI size on the approximate decoding performance and show that the optimal size of PSI can be determined based on performance requirements of applications. The proposed approach has been tested in an illustrative example of data collection in sensor networks. The simulation results confirm the benefits of approximate decoding for inter-dependent sources and further show that $93.3 \%$ of decoding errors are eliminated when the approximate decoding uses appropriate PSI.
\end{abstract}

(c) 2015 Elsevier B.V. All rights reserved.

\section{Introduction}

The hardware development of sensors and communication chipsets has enabled easy deployment of sensor networks and it has led to an excessive network traffic and demands to increase network capacity. Network coding [1] has been proposed in order to increase the throughput of networks; it can reach the max-flow capacity between the source and each destination node [2-4]. In this case, unlike

E-mail addresses: minhae.kwon@ewhain.net (M. Kwon), hyunggon.park@ewha.ac.kr (H. Park),nthomos@essex.ac.uk (N. Thomos), pascal.frossard@epfl.ch (P. Frossard). simple data forwarding in conventional networks, intermediate network nodes combine the received packets with basic coding operations. Network coding can lead to efficient resources usage (e.g., bandwidth and power), reduced computations, and improved robustness against network dynamics [5] by exploiting the diversity in networks. A variety of applications have been developed by taking advantages of network coding (e.g., content distribution, storages, and P2P systems [6-10]). Random linear network coding (RLNC) [11] is the most popular network coding algorithm, as it permits distributed deployment in dynamic error-prone networks [12]. 
Fig. 1. Overall description of system setup. At the $h$-th coding stage, the incoming $T$ innovative packets $y_{m}^{(i)}(h)(1 \leq m \leq T)$ are combined based on RLNC and $K$ outgoing packets are generated. If $T$ innovative packets are not available for the decoder at the moment of decoding (i.e., $K_{\text {in }}<T$ ), the proposed approximate decoding is deployed with side information $\nu$, which is delivered from the encoder. These are discussed in Sections 2.2 and 2.3 , respectively.

While several advantages can be obtained by deploying network coding techniques for information delivery, it has a significant drawback in practice, which is also known as all-or-nothing problem, i.e., a terminal node cannot recover any information from the received data unless it receives at least the same number of innovative coded packets ${ }^{1}$ as the number of source packets. In other words, under the conventional decoding process (e.g., Gaussian elimination), the received packets have to form a full-rank system for decoding. However, perfect decoding might not always be necessary and approximate reconstruction may be sufficient for several services that can accept imperfect reconstruction.

In order to solve this problem, we propose an approach to approximately recover inter-dependent sources from a set of network coded data that does not form a full rank system at receiver. With this same objective, a low complexity approximate decoding algorithm has been presented in [14], where the receiver simply matches the most similar data between neighbor sources and thus reaches only limited approximate decoding performance. In this paper, we present an improved approximate decoding algorithm that exploits the source characteristics, i.e., the distribution of differences between neighbor source vectors, thereby explicitly considering more general types of source data. We propose to use the mode of the distribution (i.e., the value that appears most often in a distribution) in the source characteristics to build an approximate decoding algorithm. The mode of the distribution is referred to as similarity information (SI). We show that it is sufficient side information to maximize performance of the proposed approximate decoding. As a result, the mode-based approximate decoding can significantly reduce the amount of side information needed for decoding. The decoding performance can be further improved by considering the positions where errors may occur, which are explicitly captured by the position similarity information (PSI) at the expense of additional side information. We investigate the tradeoff between the PSI size (i.e., the amount of side information or communication overheads) and the corresponding decoding

\footnotetext{
${ }^{1}$ A packet is innovative for a node if its coding vector is not in the span of the coding vectors of the packets already available at the node [13].
}

performance and show that there is an optimal amount of additional information for approximate decoding. Finally, the proposed approach is deployed in an illustrative example of sensor networks and the simulation results confirm our theoretical performance study.

The main contributions of the paper can be summarized as follows:

- we propose a generalized framework of approximate decoding that covers large range of source types,

- we develop an algorithm that enables the approximate decoding solution to be deployed for any linearly interdependent sources,

- we develop decoding algorithms that can exploit both SI and PSI, leading to significantly improved decoding performance,

- we analytically study the tradeoff between communication overhead (incurred by deploying SI and PSI) and decoding performance gains, and

- we have extensive set of experiment results that confirm the theoretical analysis.

The rest of the paper is organized as follows. The general network coding framework is presented in Section 2. The mode-based decoding approximate decoding algorithm that considers inter-dependent source distributions is proposed and discussed in Section 3. In Section 4, we show that the decoding performance can be improved by incorporating PSI into the mode-based approach. In Section 5, we evaluate and compare the performance of the mode-based approach against conventional decoding methods in an illustrative sensor network scenario. Related works are discussed in Section 6 and conclusion is drawn in Section 7.

\section{System setup}

We consider data transmission over error-prone networks that consist of source nodes, intermediate nodes and receivers. The source data is delivered to the receivers through intermediate nodes that are able to perform network coding, similar to the frameworks in [15-17]. The overview of the proposed system is shown in Fig. 1 and the details will be discussed next. 


\subsection{Source description}

Consider $T$ source vectors $\mathbf{s}_{t}(1 \leq t \leq T)$ and let $\mathbf{s}_{t}$ be the $t$ th measured symbol vector that consists of $L$ symbols denoted by $s_{t}^{(1)}, s_{t}^{(2)}, \ldots, s_{t}^{(L)}$. These symbols are discrete, i.e., $s_{t}^{(i)} \in \mathbb{R}$ for $1 \leq i \leq L$, and inter-dependent. $\mathbb{R}$ represents the field of real numbers. Since the network coding operations are performed in the Galois field $(G F)$, each $s_{t}^{(i)}$ has to be discretized and mapped into an element in GF. This operation is represented by a function $\mathcal{Q}$ that transforms the source data to $G F$ with size $2^{M}$, denoted by $G F\left(2^{M}\right)$, where $M$ is a positive integer. The function $\mathcal{Q}: \mathbb{R} \rightarrow G F\left(2^{M}\right)$ is defined as

$\mathcal{Q}\left(s_{t}^{(i)}\right)=x_{t}^{(i)} \in \mathcal{X}$

where $x_{t}^{(i)}$ is the $G F$ representation of $s_{t}^{(i)}$ in its alphabet set $\mathcal{X}$. Note that the GF size is determined by $M$ that can be appropriately chosen by considering the sizes of the source alphabet, maximum bandwidth constraints, etc. Then, the measured symbol vector $\mathbf{s}_{t}=\left[s_{t}^{(1)}, \ldots, s_{t}^{(L)}\right]^{T}$ is mapped into $\mathbf{x}_{t}=\left[x_{t}^{(1)}, \ldots, x_{t}^{(L)}\right]^{T}$ by $\mathcal{Q}$, where the notation $(\cdot)^{T}$ represents the matrix transpose operator. In order to simplify the notations used in this paper, the field of operands is implicitly assumed to be the same as the field of operators. For example, if an operator is in a $G F$, all the associated operands are implicitly assumed to be elements in the GF. The operators $\oplus$ and $\otimes$ denote the addition and multiplication in $G F$, respectively, and the operator $\odot$ represents the multiplication between matrices in GF. In this paper, $\oplus$ in $G F$ is performed by the logical bitwise exclusive-OR (XOR) operator. Conversely, if an operator is in $\mathbb{R}($ e.g.,,,$+- \times)$, the operands are also in $\mathbb{R}$.

\subsection{RLNC based encoding}

In the network, an intermediate node deploys RLNC and generates coded packets. Let $\mathbf{y}^{(i)}(h)=\left[y_{1}^{(i)}(h), \ldots\right.$, $\left.y_{m}^{(i)}(h), \ldots, y_{T}^{(i)}(h)\right]^{T}$ be incoming packets at the $h$-th coding stage; $\mathbf{y}^{(i)}(1)=\left[x_{1}^{(i)}, \ldots, x_{T}^{(i)}\right]^{T}$ is the initial source data packet. At the $h$-th coding stage intermediate node, the first $T$ innovative packets (e.g., $y_{m}^{(i)}(h)$ for $1 \leq m \leq T$ ) are combined using RLNC and generate $K$ outgoing packets (e.g., $y_{k}^{(i)}(h+1)$ for $\left.1 \leq k \leq K\right)$ as

$\mathbf{y}^{(i)}(h+1)=\mathbf{c}(h) \odot \mathbf{y}^{(i)}(h)$

or equivalently,

$\left[\begin{array}{c}y_{1}^{(i)}(h+1) \\ \vdots \\ y_{k}^{(i)}(h+1) \\ \vdots \\ y_{K}^{(i)}(h+1)\end{array}\right]=\left[\begin{array}{ccccc}c_{11}(h) & \cdots & c_{1 m}(h) & \cdots & c_{1 T}(h) \\ \vdots & \ddots & \vdots & \ddots & \vdots \\ c_{k 1}(h) & \cdots & c_{k m}(h) & \cdots & c_{k T}(h) \\ \vdots & \ddots & \vdots & \ddots & \vdots \\ c_{K 1}(h) & \cdots & c_{K m}(h) & \cdots & c_{K T}(h)\end{array}\right]$

$$
\odot\left[\begin{array}{c}
y_{1}^{(i)}(h) \\
\vdots \\
y_{m}^{(i)}(h) \\
\vdots \\
y_{T}^{(i)}(h)
\end{array}\right] \triangleq \sum_{m=1}^{T} \oplus\left\{\mathbf{c}_{m}(h) \otimes y_{m}^{(i)}(h)\right\} .
$$

The number of outgoing packets $K$ is chosen larger than $T$ (i.e., the number of symbols combined in a packet) and $K$ may depend on the expected packet erasure rate; higher $K$ is recommended for higher erasure rate and vice versa. In terms of element-wise operations, $y_{k}^{(i)}(h+1)$ is generated as

$$
\begin{aligned}
& y_{k}^{(i)}(h+1)=\sum_{m=1}^{T} \oplus\left\{c_{k m} \otimes y_{m}^{(i)}(h)\right\}=\left\{c_{k 1}(h) \otimes y_{1}^{(i)}(h)\right\} \oplus \cdots \\
& \oplus\left\{c_{k m}(h) \otimes y_{m}^{(i)}(h)\right\} \oplus \cdots \oplus\left\{c_{k T}(h) \otimes y_{T}^{(i)}(h)\right\} .
\end{aligned}
$$

Each outgoing packet $y_{k}^{(i)}(h+1)$ is a linear combination of the incoming packets $y_{m}^{(i)}(h)$ for $1 \leq m \leq T$ with coding coefficients $c_{k m}(h)$. The number of combined symbols is denoted by $T$ and $\mathbf{c}_{m}(h)$ is a coding coefficient vector that is defined as

$\mathbf{c}_{m}(h)=\left[c_{1 m}(h), \ldots, c_{k m}(h), \ldots, c_{K m}(h)\right]^{T}$.

In this paper, network coding is implemented with RLNC so that the coding coefficients are uniformly and randomly chosen from $G F\left(2^{M}\right)$, i.e., $c_{k m}(h) \in G F\left(2^{M}\right)$.

We finally note that the coded packet at the $h$-th coding stage of the network in (2) can be expressed as

$$
\begin{aligned}
& \mathbf{y}^{(i)}(h+1)=\mathbf{c}(h) \odot \mathbf{y}^{(i)}(h)=\mathbf{c}(h) \odot \mathbf{c}(h-1) \odot \cdots \odot \mathbf{c}(1) \\
& \odot \mathbf{x}^{(i)}=\mathbf{C}(h) \odot \mathbf{x}^{(i)}
\end{aligned}
$$

where $\mathbf{C}(h)$ is referred to as a global coding coefficient matrix [13], which is included in the header of the packet and delivered to the decoder to enable decoding and reconstruction. $\mathbf{c}(h)$ can be selected such that $\mathbf{C}(h)$ is invertible with high probability in RLNC [11].

In the next section, we focus on designing an approximate decoding approach, which can be deployed if a rank deficient system of equation is available at the receiver. Note that the system becomes rank deficient because of the packet loss and delay, but not because of the random selection of $\mathbf{c}(h)$ (as $\mathbf{C}(h)$ is assumed to be full-rank).

\subsection{Data reconstruction based on approximate decoding}

With the coding procedure described above, $K$ coded packets are generated at intermediate network nodes using $T$ innovative incoming packets $(K \geq T)$ and traverse error-prone network toward the destination node $h_{D}$. When a decoder receives $K_{\text {in }}$ innovative packets, ${ }^{2}$ it attempts to recover the source data $\hat{x}^{(i)}$. In the decoding process, well-known Gaussian elimination in the considered GF [18] is employed for matrix inversion and if the $K_{\text {in }} \times T$ global coding coefficient matrix $\mathbf{C}\left(h_{D}-1\right)$ is fullrank (i.e., $K_{i n}=T$ ), then $\hat{\mathbf{x}}^{(i)}=\left[\hat{x}_{1}^{(i)}, \ldots, \hat{x}_{T}^{(i)}\right]^{T}$ is uniquely determined by

$\hat{\mathbf{x}}^{(i)}=\left[\hat{x}_{1}^{(i)}, \ldots, \hat{x}_{T}^{(i)}\right]^{T}=\mathbf{C}\left(h_{D}-1\right)^{-1} \odot \mathbf{y}^{(i)}\left(h_{D}\right)$

because the inverse of a full-rank global coding coefficient matrix $\mathbf{C}\left(h_{D}-1\right)^{-1}$ is unique.

If the number of received packets is insufficient to determine $\mathbf{C}\left(h_{D}-1\right)^{-1}$ uniquely, i.e, in the presence of a singular matrix $\mathbf{C}\left(h_{D}-1\right)$ matrix (e.g., due to packet loss or

${ }^{2}$ Note that $K_{i n} \leq K$ and it depends on packet erasure of network condition. 
Summary of Notations.
Result vector of $\mathbf{x}_{t} \oplus \mathbf{x}_{t+1}$ $n$-th candidate for $\Delta$ in $G F$ Random variable for $\Delta_{n}$ Galois field with size of $2^{M}$

$\begin{array}{ll}L & \text { Length of a source vector } \\ \mathbf{c}(h) & \text { Coding coefficient matrix (with size } K \times T \text { ) at the } h \text {-th }\end{array}$ coding stage

$c_{k m}(h) \quad k$-th element of $\mathbf{c}_{m}(h)$

$\mathbf{C}(h) \quad$ Global coding coefficient matrix delays) with $K_{\text {in }}<T$, the receiver is not able to find the inverse of the coding coefficient matrix and there are multiple solutions to the system in (3). With additional constraints, a good but not-necessarily optimal solution can still be determined. In order to add constraints and find a unique solution, the idea of an approximate decoding approach is introduced in [14] that considers a simple source model and permits approximate reconstruction of sources from an insufficient number of received packets. The approximate decoding method adds simple constraints in order to form a full rank coding coefficient matrix, thereby enabling the decoding process from the system in (3) with external information. In particular, the inter-dependence between the input data is exploited in order to build additional constraints $\mathbf{D}$ and $\nu$ which render the system in (4) solvable:

$\hat{\mathbf{x}}^{(i)}=\left[\begin{array}{c}\mathbf{C}\left(h_{D}-1\right) \\ \mathbf{D}\end{array}\right]^{-1} \odot\left[\begin{array}{c}\mathbf{y}^{(i)}\left(h_{D}\right) \\ \nu\end{array}\right]$.
The coefficients in $\mathbf{D}$ and $\nu$ are in GF and depend on the source model. In this paper, $\nu$ is assumed to be delivered with high reliability. For example, separate channels such as control channels can be used [19-22] or high level of error protection techniques can be deployed for $\nu$.

A simple implementation of the approximate decoding method is presented in [14]. In particular, the matrix $\mathbf{D}$ of size $\left(T-K_{i n}\right) \times T$ is constructed such that each of its rows consists of zeros (i.e., additive identity of $G F\left(2^{M}\right)$ ) except for two elements with value " $1 " 3$ that correspond to the positions of the $x_{t}^{(i)}$ and $x_{t^{\prime}}^{(i)}$ such that $x_{t}^{(i)}=x_{t^{\prime}}^{(i)}$ in the received packets. Then, the vector $\nu$ is determined accordingly as a zero vector with size $\left(T-K_{i n}\right)$ (i.e., $\nu=\mathbf{0}_{T-K_{\text {in }}}$ ). This means that $x_{t}^{(i)}$ and $x_{t^{\prime}}^{(i)}$ shall have the same value in the approximate decoding algorithm proposed in [14]. Finally, an approximation $\hat{\mathbf{x}}^{(i)}$ of the original data is

\footnotetext{
${ }^{3}$ Recall that 1 is also an additive inverse of 1 in $G F\left(2^{M}\right)$.
}

obtained as

$\hat{\mathbf{x}}^{(i)}=\left[\begin{array}{c}\mathbf{C}\left(h_{D}-1\right) \\ \mathbf{D}\end{array}\right]^{-1} \odot\left[\begin{array}{l}\mathbf{y}^{(i)}\left(h_{D}\right) \\ \mathbf{0}_{\left(T-K_{\text {in }}\right)}\end{array}\right]$

where $\mathbf{0}_{\left(T-K_{i n}\right)}$ is a vector with $\left(T-K_{i n}\right)$ zeros. This approach permits the receiver to approximately reconstruct the original symbols when the number of symbols is not sufficient for perfect decoding.

The key idea of the approximate decoding method is to incorporate additional equations (i.e., D) based on source model, such that the matrix $\left[\mathbf{C}\left(h_{D}-1\right)^{T} \mathbf{D}^{T}\right]^{T}$ in (5) becomes full rank. Without changes in conventional decoding algorithm (e.g., Gaussian elimination), approximate decoding algorithm does not need complex processes such as belief propagation [23] and it can be easily used when the received data does not form a full rank system. While the approximate decoding algorithm in [14] shows a new paradigm of network coded data reconstruction, the additional equations in (5) are very simple and cannot fully capture complex inter-dependent source models. Therefore, the algorithm in [14] can be used only in limited source model. In order to overcome the limitation, we propose an improved algorithm for approximate decoding that explicitly considers the statistical characteristics of the sources.

For reader's convenience, we summarize notations and abbreviations frequently used in this paper in Tables 2 and 3 , respectively.

\section{Mode-based approximate decoding for inter- dependent sources}

In this section, we develop a new approximate decoding algorithm that explicitly considers the source characteristics for data reconstruction with incomplete sets of received packets. 


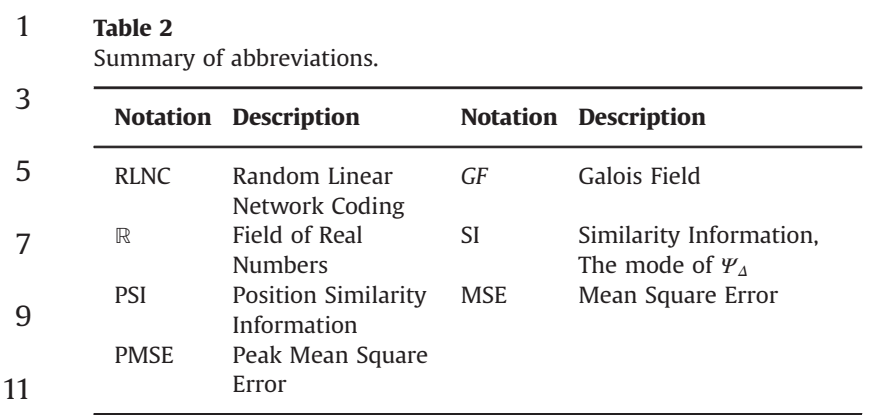

\subsection{Description of inter-dependent source distribution}

We consider a set of inter-dependent discrete sources. The sources are characterized by the distribution (i.e., probability mass function) of the difference between values in source data vectors. Let $\delta_{t}=\left[\delta_{t}^{(1)}, \ldots, \delta_{t}^{(L)}\right]^{T}$ be an $L^{-}$ element vector representing the difference between data of the $t$-th and ( $t+1)$-th sources, i.e., $\mathbf{x}_{t}$ and $\mathbf{x}_{t+1}$, respectively, and $\delta_{t}^{(i)}$ be the $i$-th element of $\boldsymbol{\delta}_{t}$. We assume that the elements in $\boldsymbol{\delta}_{t}$ follow a unimodal distribution $\Psi_{\Delta}$ where its mode is $\Delta$. Here, the source data can be expressed as

$\mathbf{x}_{t+1}=\mathbf{x}_{t}+\boldsymbol{\delta}_{t}$

where $\delta_{t}^{(i)} \sim \Psi_{\Delta}$, or equivalently,

$x_{t+1}^{(i)}=x_{t}^{(i)}+\delta_{t}^{(i)} \quad$ for all $1 \leq i \leq L$.

In order to describe the entire source vectors, a single or multiple $\Psi_{\Delta}$ can be deployed. The inter-dependent source model can be exploited in a wide range of applications such as brightness changes in pictures, temperature variances, and seismic signals at different sensors [24,25]. The unimodal distribution can further represent noise in source data. A unimodal distribution includes various stochastic models such as Gaussian, Laplacian, chi-square and Cauchy distribution. An illustrative example of the above source model is shown in Fig. 2.

\subsection{Approximate decoding algorithm for inter-dependent sources}

We now discuss how to design the constraints $\nu=\left[\nu_{1}, \nu_{2}, \ldots, \nu_{T-K_{i n}}\right]^{T}$ that complete the decoding system in (4) such that the performance of the approximate decoding is maximized given the inter-dependent source distribution $\Psi_{\Delta}$. The constraints $\nu$ can provide additional equations that render the underdetermined decoding system solvable. Since the inter-dependent sources are characterized by $\delta_{t}^{(i)} \sim \Psi_{\Delta}$, $\nu$ needs to be designed such that it represents the source characteristics.

Let $\nu^{*}$ be the optimal constraints $\nu$ that lead to a perfect decoding in (4). It is therefore desired that $\nu=\boldsymbol{\nu}^{*}$. If $\nu \neq \boldsymbol{\nu}^{*}$, the more zeros in the vector of $\boldsymbol{\nu}-\boldsymbol{\nu}^{*}$, the better performance of approximate decoding algorithm [17]. An illustrative result is shown in Fig. 3, where source data vectors have inter-dependency parameterized with $\nu_{n}^{*}=16$, i.e., $x_{t+1}^{(i)}=x_{t}^{(i)}+16$ and RLNC is deployed in $G F\left(2^{10}\right)$. The decoding is based on Gaussian elimination and the decoding performance is measured by Peak Mean Square
Error (PMSE) [26] for various $\nu=\nu_{n} \cdot \mathbf{1}_{\left(T-K_{i n}\right)}$ in (4). The average PMSE for 1000 independent experiments is shown in Fig. 3, where the source vector $\mathbf{x}_{1}$ is randomly and independently determined in every experiment. This result confirms that approximate decoding leads to the best performance only when $\nu_{n}^{*}=\nu$ among $1 \leq \nu_{n} \leq 32$. Contrary to the intuition that closeness of $\nu_{n}$ to $\nu_{n}^{*}$ would increase the decoding performance, there is no obvious relationship between $\nu_{n}^{*}-\nu_{n}$ and performance. Rather, the number of zeros in $\boldsymbol{\nu}-\boldsymbol{\nu}^{*}$ determines the performance of approximate decoding. ${ }^{4}$ The analysis of the decoding performance behavior is presented in Appendix A.

The shape of decoding performance given $\nu_{n}^{*}$ (see Fig. 3 and Appendix A) is well captured by a cost model $\mathcal{C}\left(\nu_{n} ; \nu_{n}^{*}\right)$, referred to as the Cauchy-Dirac delta function [27], defined as

$\mathcal{C}\left(\nu_{n} ; \nu_{n}^{*}\right)=\lim _{\gamma \rightarrow 0}\left(1-f\left(\nu_{n} ; \nu_{n}^{*}, \gamma\right)\right)= \begin{cases}0 & \text { if } \nu_{n}=\nu_{n}^{*} \\ 1 & \text { otherwise }\end{cases}$

where the probability density function of the Cauchy distribution is given by

$f\left(\nu_{n} ; \nu_{n}^{*}, \gamma\right)=\frac{1}{\pi \gamma\left[1+\left(\frac{\nu_{n}-\nu_{n}^{*}}{\gamma}\right)^{2}\right]}$

Here, $\nu_{n}^{*}$ and $\gamma$ are determined by the sources as $\nu_{n}^{*}$ denotes the parameter that specifies the position of the peak of the distribution and $\gamma$ is a scale parameter that specifies the half-width at half-maximum. Since $f\left(\nu_{n} ; \nu_{n}^{*}, \gamma\right)$ is a probability measure, $f\left(\nu_{n} ; \nu_{n}^{*}, \gamma\right) \in[0,1]$. Therefore, the expected cost, which represents the decoding error, with any interdependent source distribution $\Psi_{\Delta}$, is expressed as

$E\left\{\mathcal{C}\left(\nu_{n} ; \nu_{n}^{*}\right)\right\}=\sum_{\nu_{n} \in \nu} \mathcal{C}\left(\nu_{n} ; \nu_{n}^{*}\right) \operatorname{Pr}\left(V_{n}=\nu_{n}\right)$

where $V_{n}$ is a random variable for $\nu_{n} \in \nu$ and is characterized by $\Psi_{\Delta}$. In Property 1 , we show that SI (i.e., $\Delta$ ) leads to the minimum expected cost given the source distribution $\Psi_{\Delta}$.

Property 1. Given the inter-dependent source distribution, the mode of distribution (SI) minimizes expected cost of approximate decoding.

Proof. The goal of the proof is to show that $\left(\nu_{n}^{*}\right)_{o p t}=\Delta$ is the minimizer of the expected cost. The corresponding optimization problem is given by

$\left(\nu_{n}^{*}\right)_{\text {opt }}=\arg \min _{\nu_{n}^{*} \in \nu} E\left\{\mathcal{C}\left(\nu_{n} ; \nu_{n}^{*}\right)\right\}$

$\left(\nu_{n}^{*}\right)_{o p t}=\arg \min _{\nu_{n}^{*} \in \nu} \sum_{\nu_{n} \in \nu} \mathcal{C}\left(\nu_{n} ; \nu_{n}^{*}\right) \operatorname{Pr}\left(V_{n}=\nu_{n}\right)$

$\left(\nu_{n}^{*}\right)_{o p t}=\arg \min _{\nu_{n}^{*} \in \nu_{\nu_{n}} \in \boldsymbol{\nu}, \nu_{n} \neq \nu_{n}^{*}} \operatorname{Pr}\left(V_{n}=\nu_{n}\right)$

$\left(\nu_{n}^{*}\right)_{o p t}=\arg \min _{\nu_{n}^{*} \in \nu}\left[1-\operatorname{Pr}\left(V_{n}=\nu_{n}^{*}\right)\right]$

\footnotetext{
${ }^{4}$ Note that PMSE for $\nu_{n}=\nu_{n}^{*}$ is not zero in this experiment result. This is because $\nu=\nu_{n}^{*} \cdot \mathbf{1}_{\left(T-K_{i n}\right)}$ is not enough for perfect decoding in GF where network coding operations are performed, while it can provide enough information in $\mathbb{R}$. This is studied in Property 3.
} 


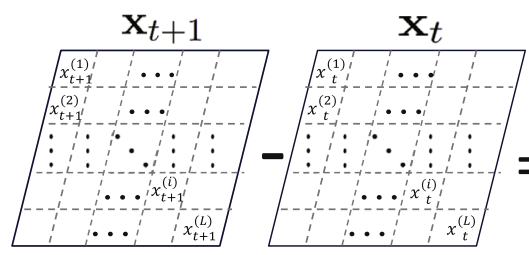

$$
\begin{aligned}
& \mathbf{x}_{t+1}=\mathbf{x}_{t}+\boldsymbol{\delta}_{t} \\
& x_{t+1}^{(i)}=x_{t}^{(i)}+\delta_{t}^{(i)}
\end{aligned}
$$

Fig. 2. An illustrative example of matrix-shaped inter-dependent sources and corresponding difference $\delta_{t}$. $\delta_{t}^{(i)}$ is an element of $\delta_{t}$ and $\delta_{t}^{(i)}$ follows a unimodal distribution $\Psi_{\Delta}$ where its mode is $\Delta$.

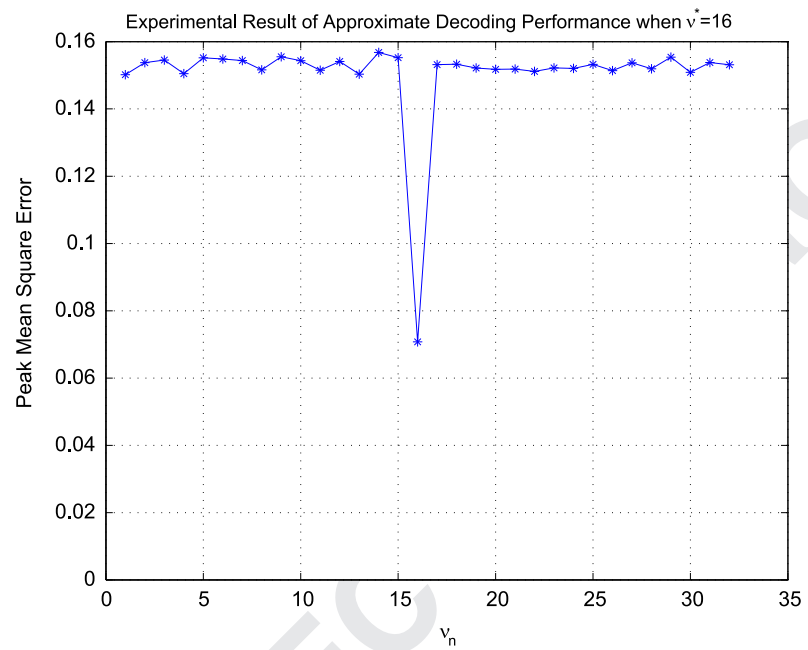

Fig. 3. An illustrative example for the average performance of approximate decoding with various $\nu_{n}$ when $\nu_{n}^{*}=16$. The performance of approximate decoding is maximized only if $\nu_{n}=\nu_{n}^{*}=16$. In this example, $G F\left(2^{10}\right)$ is used and the sources are randomly and independently determined over 1000 times.

$$
\left(\nu_{n}^{*}\right)_{o p t}=\arg \max _{\nu_{n}^{*} \in \nu} \operatorname{Pr}\left(V_{n}=\nu_{n}^{*}\right)
$$

$\left(\nu_{n}^{*}\right)_{o p t}=\Delta$.

The equality between (12) and (13) comes from the definition of cost function given in (8), i.e., $\mathcal{C}\left(\nu_{n} ; \nu_{n}^{*}\right)=1$ if $\nu_{n} \neq \nu_{n}^{*}$ and $\mathcal{C}\left(\nu_{n} ; \nu_{n}^{*}\right)=0$ if $\nu_{n}=\nu_{n}^{*}$. Since $\operatorname{Pr}\left(V_{n}=\nu_{n}\right) \geq 0$, the minimization of $1-\operatorname{Pr}\left(V_{n}=\nu_{n}^{*}\right)$ is equivalent to the maximization of $\operatorname{Pr}\left(V_{n}=\nu_{n}^{*}\right)$, leading to (14). Since $V_{n}$ is characterized by the inter-dependent source distribution $\Psi_{\Delta}$, $\max \operatorname{Pr}\left(V_{n}=\nu_{n}^{*}\right)$ is achieved only if $\nu_{n}^{*}=\Delta$. Therefore, $\left(\nu_{n}^{*}\right)_{\text {opt }}=\Delta$ minimizes the expected cost. $\square$

The above property means that the decoder only needs to know $\Delta$ instead of the entire distribution $\Psi_{\Delta}$ in order to have effective decoding; the amount of the additional information that needs to be delivered is minimized.

This property is confirmed by the simulation results shown in Fig. 4. To generate an illustrative set of linearly inter-dependent source data (i.e., $x_{t+1}^{(i)}=x_{t}^{(i)}+\delta_{t}^{(i)}$ ), the source dependency $\delta_{t}^{(i)}$ is generated based on a Gaussian distribution $\mathcal{N}\left(16, \sigma^{2}\right)$ in Fig. 4(a), where the mean is 16 and the variance is $\sigma^{2}$. For a Gaussian distribution, the mode is the same as the mean, i.e., $\mathrm{SI}=16(\Delta=16)$. For the sources in Fig. 4(b), $\delta_{t}^{(i)}$ is generated based on the Laplacian distribution $\mathcal{L}\left(16, \sigma^{2} / 2\right)$ where the location parameter is 16 and the scale parameter is $\sigma^{2} / 2$. For the Laplacian distribution, the mode is the same as the location parameter, i.e., $\mathrm{SI}=16(\Delta=16)$. Hence, $\mathrm{SI}=\arg \max _{\nu_{n}} \operatorname{Pr}\left(V_{n}=\nu_{n}\right)=16$ in both cases. In these simulations, three source data vectors are combined together based on RLNC and 1 out of 3 packets is lost (i.e., 1/3 packet loss rate) so that approximate decoding strategies are deployed in $G F\left(2^{8}\right)$. The results are given in average PMSE computed from 1000 independent experiments. For the sake of comparison, we consider the following alternative three strategies [17]:

- Strategy 1 (S1): Select a single value $\Delta$ in $\Psi_{\Delta}$ (i.e., SI) as shown in Property 1, i.e., $\nu=\Delta \cdot \mathbf{1}_{\left(T-K_{i n}\right.}$.

- Strategy 2 (S2): Sample the inter-dependent source distribution $\Psi_{\Delta}$ to select values of $\nu_{n}$, i.e., $\nu_{n} \sim \Psi_{\Delta}$.

- Strategy 3 (S3): Sample a uniform distribution $\mathcal{U}\left(0,2^{8}-1\right)$ to select values of $\nu_{n}$, i.e., $\nu_{n} \sim \mathcal{U}\left(0,2^{8}-1\right)$.

From the results, it is obvious that average PMSE is minimized by selecting SI (i.e., Strategy 1), which confirms Property 1.

In summary, under the knowledge of inter-dependent source distribution, SI for $\nu$ leads to the minimized expected $\operatorname{cost}\left(\right.$ i.e., $\left.\nu=\Delta \cdot \mathbf{1}_{\left(T-K_{\text {in }}\right)}\right)$. More importantly, it implies that the performance of the approximate decoding algorithm 
a

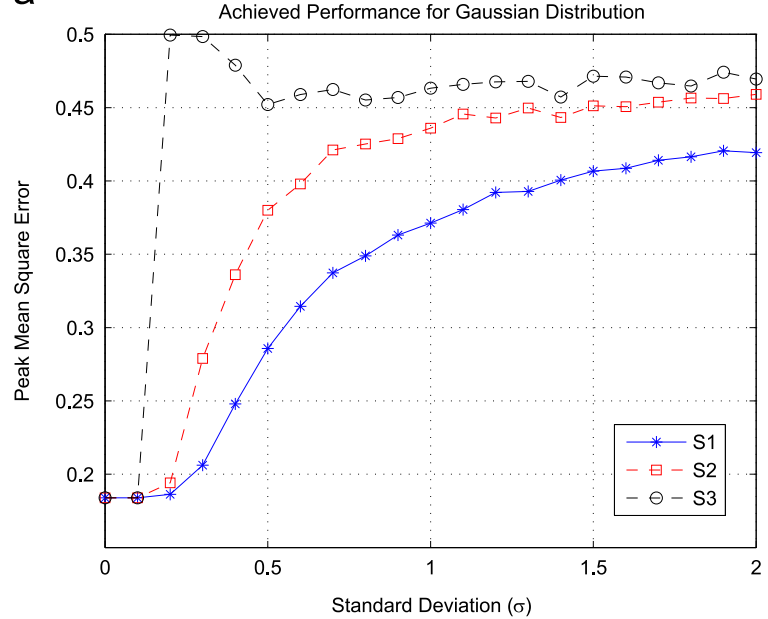

b

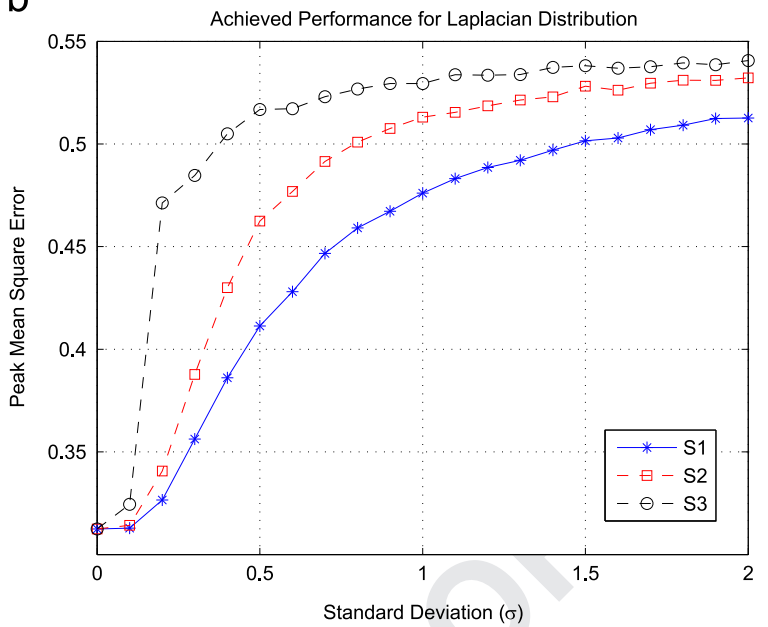

63 only $\Delta$ but also the other outcomes. For example, when sources are described as $\mathbf{x}_{t}-\mathbf{x}_{t+1}=\Delta=16$ in $\mathbb{R}, \mathbf{x}_{t} \oplus \mathbf{x}_{t+1}$ in $G F$ includes not only 16 but also other values such as 48 , $112, \ldots$, in $G F\left(2^{8}\right)$. Hence, we define $\boldsymbol{\Delta}_{t}$ as the vector of outcomes from $\mathbf{x}_{t+1} \oplus \mathbf{x}_{t}$, i.e., $\boldsymbol{\Delta}_{t}=\mathbf{x}_{t+1} \oplus \mathbf{x}_{t}$ where each element in $\boldsymbol{\Delta}_{t}$ is denoted by $\Delta_{n}$. These elements are referred to as candidates of $\Delta$. Since there may be different occurrences of $\Delta_{n}$ in $\Delta_{t}$, we consider $\Delta_{n}$ as a random variable, is denoted by $\Delta_{R}$.

As will be discussed later, $\operatorname{Pr}\left(\Delta_{R}=\Delta_{n}\right)$ is decreasing with respect to $n$. This means that the probability of $\Delta_{1}$ is the highest among the candidates of $\Delta$ and $\Delta_{1}=\Delta$ by definition. This is illustrated in Table 3 and the details are discussed in Property 3. Moreover, as discussed in Section 3.2, the accurate positions (or indices) of the candidates are the key information for improved performance of the proposed approximate decoding framework. Therefore, Strategy 1 (S1) in Section 3.2, which is shown to be the best implementation of approximate decoding for unimodal inter-dependent sources, can be further improved by considering the positions of the candidates $\boldsymbol{\Delta}_{t}$ included in PSI.

The PSI includes $\Delta_{1}$ by default as it has the highest probability of occurrence in $\Delta_{t}$. Then, it includes the position indices of $\Delta_{n}$ in $\Delta_{t}$ for $n \geq 2$. The $n$-th row of PSI is filled with position indices of $\Delta_{n+1}$ in $\Delta_{t}$. An illustrative example of PSI construction is shown in Fig. 5. If a decoder receives PSI, Strategy 1 (S1) that is based only on $\Delta$ is deployed as the baseline of approximate decoding, i.e., $\boldsymbol{\Delta}_{P S I}=\Delta_{1} \cdot \mathbf{1}_{\left(T-K_{i n}\right)}$. Next, the elements with the position indices that are written in $n$-th row of PSI are replaced by $\Delta_{n+1}$ in $\boldsymbol{\Delta}_{P S I}$. Finally, $\boldsymbol{\nu}$ in (4) is replaced by $\boldsymbol{\Delta}_{P S I}$, leading to

$\hat{\mathbf{x}}^{(i)}=\left[\begin{array}{c}\mathbf{C}\left(h_{D}-1\right) \\ \mathbf{D}\end{array}\right]^{-1} \odot\left[\begin{array}{c}\mathbf{y}^{(i)}\left(h_{D}\right) \\ \boldsymbol{\Delta}_{P S I}\end{array}\right]$.

In the next section, we study the properties of proposed approximate decoding with PSI that helps us to increase performance. 
4.2. Properties of approximate decoding with psi for $\Delta$-lin-

\begin{tabular}{|c|c|}
\hline $\mathbf{x}_{\mathbf{t}}$ & $\mathbf{x}_{\mathbf{t}+\mathbf{1}}$ \\
\hline 143 & 159 \\
\hline 355 & 371 \\
\hline 49 & 65 \\
\hline 421 & 437 \\
\hline 23 & 39 \\
\hline 162 & 178 \\
\hline 486 & 502 \\
\hline 17 & 33 \\
\hline
\end{tabular}

$\boldsymbol{\Delta}_{t}$ indices for $\Delta_{n}$ for $n \geq 2$. early inter-dependent source
In this section, we focus on the investigation of the basic properties of the proposed approximate decoding algorithm with PSI for $\Delta$-linearly inter-dependent sources. We first consider a special case where $\Delta=2^{k}$ and then generalize the case to $\Delta \neq 2^{k}$.

Property 2. For $\Delta$-linearly inter-dependent sources, the expected distortion of approximate decoding with $\Delta=2^{k}$ $(0 \leq k<M)$ is always lower than that with $\Delta^{\prime}$ such that $2^{k-1}<\Delta^{\prime}<2^{k+1}, \Delta^{\prime} \neq 2^{k}$.

Proof. Any element in $G F\left(2^{M}\right)$ can be represented by $M$-bit binary numbers. Since $\mathbf{x}_{t+1}=\mathbf{x}_{t}+\Delta \cdot \mathbf{1}$ in (16), there is no distortion if

$\left(\mathbf{x}_{t}+\Delta \cdot \mathbf{1}\right) \oplus(\Delta \cdot \mathbf{1})=\mathbf{x}_{t}$.

Let $\omega(\mathbf{x}, \mathbf{y})$ denote the number of "1" that are at the same position of binary representations of $\mathbf{x}$ and $\mathbf{y}$. Note that, the condition given in (18) is satisfied if and only if $\omega\left(\mathbf{x}_{t}, \Delta \cdot \mathbf{1}\right)=0$, because an overlap between any two "1"s at the same position of $\mathbf{x}_{t}$ and $\Delta \cdot \mathbf{1}$ results in carriage returns from $x_{t}^{(i)}+\Delta$. This leads to decoding errors (or distortion), implying that the distortion can be minimized with the smallest $\omega\left(\mathbf{x}_{t}, \Delta \cdot \mathbf{1}\right)$. Since

$\min \omega\left(x_{t}^{(i)}, \Delta\right)=1$

for all $i$, the distortion is minimized when $\Delta=2^{k}$, $0 \leq k<M$. This is equivalent to the case where only a

Table 3

Examples of candidates for $\Delta=2^{k}$ over $G F\left(2^{8}\right)$.

\begin{tabular}{lllllll}
\hline & $\Delta_{1}$ & $\Delta_{2}$ & $\Delta_{3}$ & $\Delta_{4}$ & $\Delta_{5}$ & $\Delta_{6}$ \\
\hline$\Delta=2^{2}$ & $2^{2}$ & $\sum_{k=2}^{3} 2^{k}$ & $\sum_{k=2}^{4} 2^{k}$ & $\sum_{k=2}^{5} 2^{k}$ & $\sum_{k=2}^{6} 2^{k}$ & $\sum_{k=2}^{7} 2^{k}$ \\
$\Delta=2^{3}$ & $2^{3}$ & $\sum_{k=3}^{4} 2^{k}$ & $\sum_{k=3}^{5} 2^{k}$ & $\sum_{k=3}^{6} 2^{k}$ & $\sum_{k=3}^{7} 2^{k}$ & \\
$\Delta=2^{4}$ & $2^{4}$ & $\sum_{k=4}^{5} 2^{k}$ & $\sum_{k=4}^{6} 2^{k}$ & $\sum_{k=4}^{7} 2^{k}$ & & \\
$\Delta=2^{5}$ & $2^{5}$ & $\sum_{k=5}^{6} 2^{k}$ & $\sum_{k=5}^{7} 2^{k}$ & & & \\
$\Delta=2^{6}$ & $2^{6}$ & $\sum_{k=6}^{7} 2^{k}$ & & & & \\
\hline
\end{tabular}

single bit 1 is set at the $(k+1)$-th position of the binary representation of $\Delta$, which completes the proof.

In order to construct the PSI, the candidates $\Delta_{t}$ need to be found, as discussed in Property 3. To be consistent with the assumptions on the sources, $x_{t}^{(i)}+\Delta$ does not exceed the size of $G F$, i.e., $0 \leq x_{t}^{(i)}<2^{M}-2^{k}$ such that all source data are in $G F\left(2^{M}\right)$.

Property 3. If $\Delta=2^{k}(0 \leq k<M)$ in $G F\left(2^{M}\right)$, there are at most $(M-k)$ candidates in $\boldsymbol{\Delta}_{t}$ where $n$-th candidate $\Delta_{n}$ is expressed as

$\Delta_{n}=\sum_{i=k}^{k+n-1} 2^{i}$

with the probability of

$\operatorname{Pr}\left(\Delta_{R}=\Delta_{n}\right)=\frac{2^{(M-k-n)}}{2^{(M-k)}-1}$.

79

81

Proof. An element in $G F\left(2^{M}\right)$ is represented by $M$ bits. For an element $\Delta=2^{k}$ in $G F\left(2^{M}\right)$, it can be represented by only a single bit " 1 " at the $(k+1)$-th position and " 0 " at all the other positions. Recall that the candidates are generated when

$x_{t+1}^{(i)} \oplus \Delta=\left(x_{t}^{(i)}+\Delta\right) \oplus \Delta \neq x_{t}^{(i)}$.

If bit " 1 " is set for $(k+1)$-th, $\ldots,(k+n-1)$-th position of $x_{t}^{(i)}$ when the bit " 1 " is set for both $(k+1)$-th position of $\Delta$ and $x_{t}^{(i)}, \Delta_{n}$ is generated with a value of $\Delta_{n}=\sum_{i=k}^{k+n-1} 2^{i}$. Since $\Delta_{n}$ is in $G F\left(2^{M}\right), 1<n \leq M-k$, there are at most $M-k$ candidates.

Next, we show that $\operatorname{Pr}\left(\Delta_{R}=\Delta_{n}\right)=2^{(M-k-n)} /\left(2^{(M-k)}-1\right)$. Since $x_{t}^{(i)}$ is in the range of $0 \leq x_{t}^{(i)}<2^{M}-2^{k}, x_{t}^{(i)}$ can take one of $2^{M}-2^{k}$ different values. Given a $\Delta_{n}$, there are $2^{M-n}$ different values for $x_{t}^{(i)}$ because the $(k+n)$-th position of $x_{t}^{(i)}$ is set to " 0 " and the positions of $x_{t}^{(i)}$ from $(k+n-1)$-th to $(k+1)$-th are set to " 1 ". Therefore, $\Delta_{n}$ is generated with probability

$\operatorname{Pr}\left(\Delta_{R}=\Delta_{n}\right)=\frac{2^{(M-n)}}{2^{M}-2^{k}}$

Position

Index

\begin{tabular}{|l|}
\hline$\Delta_{1}=16$ \\
\hline$\Delta_{1}=16$ \\
\hline$\Delta_{3}=112$ \\
\hline$\Delta_{1}=16$ \\
\hline$\Delta_{2}=48$ \\
\hline$\Delta_{1}=16$ \\
\hline$\Delta_{1}=16$ \\
\hline$\Delta_{2}=48$ \\
\hline
\end{tabular}

\begin{tabular}{c|c}
1 & PSI \\
\hline 2 & (Position Similarity Information)
\end{tabular}

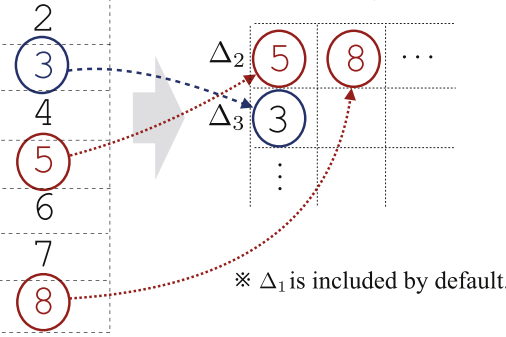

111

113

115

117

119

121

Fig. 5. An illustrative example of PSI construction. In this example, $\mathbf{x}_{t}-\mathbf{x}_{t+1}=\Delta=16$ in $\mathbb{R}$. However, $\mathbf{x}_{t} \oplus \mathbf{x}_{t+1}$ in $G F$ includes not only 16 but also other values such as $48,112, \ldots$, in $G F\left(2^{8}\right)$. The results from $\mathbf{x}_{t} \oplus \mathbf{x}_{t+1}$ are referred to as candidates of $\Delta=16$. The PSI includes $\Delta_{1}$ by default and the position 
$\operatorname{Pr}\left(\Delta_{R}=\Delta_{n}\right)=\frac{2^{(M-k-n)}}{2^{(M-k)}-1}$ for $1 \leq n \leq M-k$

which completes the proof. $\square$

The candidates $\boldsymbol{\Delta}_{t}$ and the corresponding probability mass function (PMF) based on Property 3 are shown in Table 4 when $\Delta=2^{k}$ in $G F\left(2^{M}\right)$. This enables us to construct a PSI matrix, where each row of PSI represents a candidate.

9 For example, the first row has the index numbers of $\Delta_{2}$, the second row has the index numbers of $\Delta_{3}$, and in general,

11 the $(M-n-1)$-th row has the index numbers of $\Delta_{M-n}$ for $n=1, \ldots, k$. As discussed above, while the approximate decoding with the PSI leads to improved performance, the use of PSI results in more communication overhead compared to SI. Hence, it is essential to investigate the tradeoff between the amount of overhead for transmitting PSI and the performance improvement. This tradeoff is studied in Property 4.

Property 4. If the (n-1)-th row of PSI or $\Delta_{n}$ is additionally used for approximate decoding, its performance can be improved by a factor of $1 / 2^{(n-1)}$.

23 Proof. As shown in Table $4, \operatorname{Pr}\left(\Delta_{R}=\Delta_{n}\right)$ is given by $2^{(M-k-n)} / 2^{(M-k)}-1$. This means that $\operatorname{Pr}\left(\Delta_{R}=\Delta_{n}\right)$ decreases by $1 / 2^{n}$, as $n$ increases. Thus, if $\Delta_{n}$ is additionally used for approximate decoding, the performance can be improved by $1 / 2^{(n-1)}$ times compared to the gain obtained if $\Delta_{n-1}$ is used. $\square$

The performance improvement based on additional information in PSI is interpreted as an additional refinement. Property 4 is used as a guideline for the trade-off between the communication overhead and the decoding performance of the proposed approximate decoding algorithm.

Unlike the case of $\Delta=2^{k}$, the candidates for the case of $\Delta \neq 2^{k}$ cannot be easily identified. In this case, the candidates $\Delta_{t}$ can be found by decomposing into the sum of $2^{k} \mathrm{~S}$ for $0 \leq k \leq M$ and using the candidates of $\Delta=2^{k}$. The above description is explained in Algorithm 1.

Algorithm 1. Candidates $\boldsymbol{\Delta}_{t}$.

Given: $\Delta$ and $G F\left(2^{M}\right)$.

1 : if $\Delta=2^{k}$ then

2: $\quad \Delta_{t}=\left\{\Delta_{1}, \ldots, \Delta_{n}, \ldots, \Delta_{M-k}\right\}$ where $\Delta_{n}=\sum_{i=k}^{k+n-1} 2^{i} / / \operatorname{Prop}-$ erty 3

3: else // if $\Delta \neq 2^{k}$

4: Find $p_{1}, p_{2}, \ldots, p_{n}$ such that $\Delta=\sum_{j=1}^{n} 2^{p_{j}}$ for $0 \leq p_{n}<\cdots<p_{2}<p_{1}$

5: Find all the candidates $\Delta_{t}$ based on Property 3 , if $\Delta=2^{p_{n}}$ (denoted by $\boldsymbol{\Delta}_{t}\left(2^{p_{n}}\right)$ ).

6: Compute Cartesian product set, $\prod_{l=1}^{n} \boldsymbol{\Delta}_{t}\left(2^{p_{l}}\right)=\left\{\left(\gamma_{1}, \ldots, \gamma_{n}\right) \mid \gamma_{i} \in \boldsymbol{\Delta}_{t}\left(2^{p_{i}}\right)\right\}$
7: Calculate the sum of a tuple $\Gamma_{i}=\sum_{j=1}^{n} \gamma_{j}$, where $\Gamma_{i}$ is sum of $i$ th tuple elements

8: $S=\left\{\Gamma_{1}, \Gamma_{2}, \ldots, \Gamma_{q}\right\}$, where $q=\prod_{l=1}^{n}\left|\Delta_{t}\left(2^{p_{l}}\right)\right|$ and $|\cdot|$ denotes cardinality

9: $\quad$ if there are even number of $\Gamma_{i}$ in $S$ then

11: else// odd numbers

12: if there are multiple instances of $\Gamma_{i}$ in $S$ then merge them to a single $\Gamma_{i}$

13: end if

14: $\Delta_{t} \leftarrow S$

15: end if

16: end if

\subsection{PSI properties illustrations}

We implement here our approximate decoding method in conjunction with PSI and we experimentally analyze the properties studied in the previous section. In the following simulations, we consider a set of three $\Delta$-linearly interdependent sources, which are randomly generated as in (16).

Fig. 6 shows the performance of approximate decoding with PSI, which is measured by PMSE over various packet loss rates with different amounts of additional information delivered by the PSI.

In these experiments, the first source data vector $\mathbf{x}_{1}$ is randomly generated and neighbor source vectors are generated by $\mathbf{x}_{t}=\mathbf{x}_{1}+(t-1) \cdot \Delta$ for $t=1, \ldots, T$. Hence, the sources are $\Delta$-linearly inter-dependent. For Fig. 6(a) and (b), $\Delta=8$ and $\Delta=10$ are used, respectively. Since $\Delta=10$ is not the form of $2^{k}$, candidates are found based on Algorithm 1 . Ten data vectors are combined together based on RLNC in $G F\left(2^{10}\right)$. The experiments are independently repeated 1000 times.

It is clear from Fig. 6(a) and (b) that the approximate decoding solution for different PSI outperforms the approximate decoding with $\mathrm{PSI}_{0}$ or $\mathrm{SI}$, over all the range of packet loss rates. Moreover, the approach proposed in Algorithm 1 is effective for any $\Delta$ s that are not necessarily powers of 2 . The results indicate that the additional information given by PSI needs to be adaptively structured by explicitly considering the packet loss rates and the targeted performance.

Fig. 7 confirms Property 4, which says that additional $\Delta_{n}$ can improve the performance of approximate decoding by a factor of $1 / 2^{n-1}$. For example, if $\Delta_{2}$ is used in addition to $\Delta_{1}$, the performance (measured by PMSE) improves from approximately 0.06 to 0.03 . Similarly, if $\Delta_{3}$ is used in addition to $\Delta_{1}$ and $\Delta_{2}$, the performance improves again from approximately 0.03 to 0.015 . We finally note that there is no error if $\Delta_{1}, \ldots, \Delta_{6}$ are included in PSI. This is because $\Delta_{1}, \ldots, \Delta_{6}$ are the information that can be maximally provided to the decoder (recall that $M-k=8-2=6$ for $\Delta=2^{k}=4$ and $G F\left(2^{M}\right)=G F\left(2^{8}\right)$ as discussed in Property 3$)$.
10: $\quad$ Remove $\Gamma_{i}$ from $S$

Table 4

Candidates of $\Delta$ and PMF of the candidates if $\Delta=2^{k}$ in $G F\left(2^{M}\right)$.

\begin{tabular}{|c|c|c|c|c|c|c|}
\hline & $\Delta_{1}$ & $\Delta_{2}$ & $\cdots$ & $\Delta_{n}$ & $\ldots$ & $\Delta_{M-k}$ \\
\hline$\Delta_{t}$ & $2^{k}$ & $\sum_{j=k}^{k+1} 2^{j}$ & $\cdots$ & $\sum_{j=k}^{k+(n-1)} 2^{j}$ & $\cdots$ & $\sum_{j=k}^{M-1} 2^{j}$ \\
\hline \multirow{2}{*}{$\operatorname{Pr}\left(\Delta_{R}=\Delta_{n}\right)$} & $2^{(M-k-1)}$ & $2^{(M-k-2)}$ & $\cdots$ & $2^{(M-k-n)}$ & $\cdots$ & 1 \\
\hline & $\overline{2^{(M-k)}-1}$ & $2^{(M-k)}-1$ & & $2^{(M-k)}-1$ & & $\overline{2^{(M-k)}-1}$ \\
\hline
\end{tabular}


a

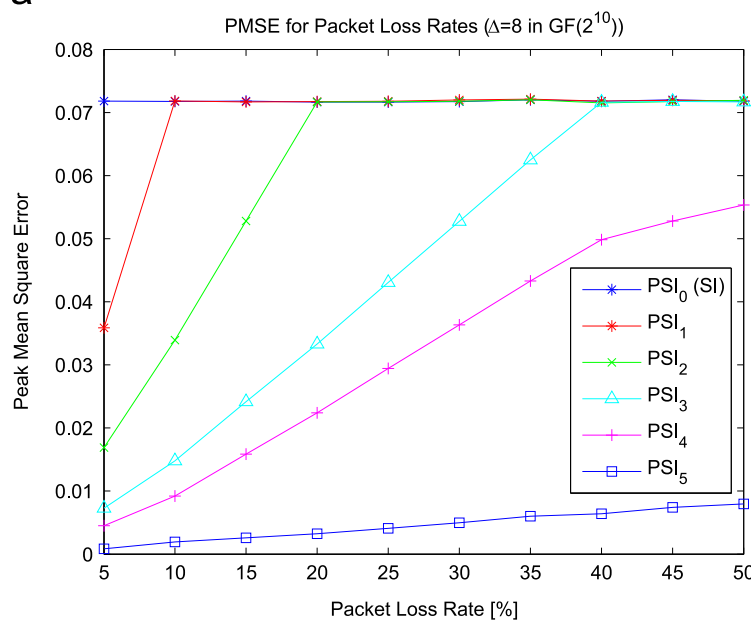

b

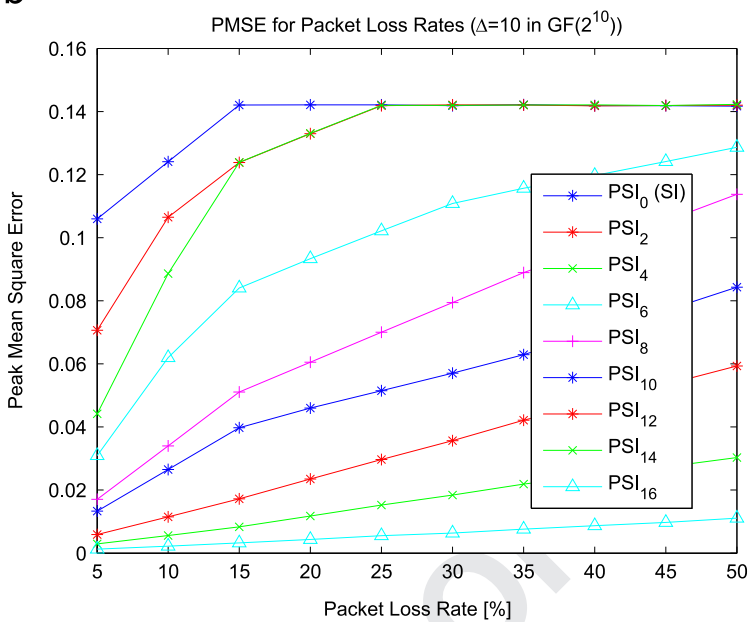

63

65

69

71

73

75

Fig. 6. Average PMSE of the proposed decoding algorithm for various sizes of PSI. In order to find candidates for $\Delta=10$, Algorithm 1 is used. PSI 0 denotes the case where only SI is delivered and $\operatorname{PSI}_{n}$ denote cases where $\left\{\Delta_{1}, \ldots, \Delta_{n}\right\}$ is included in PSI. (a) $\Delta=8, G F\left(2^{10}\right)$ and (b) $\Delta=10, G F\left(2^{10}\right)$.

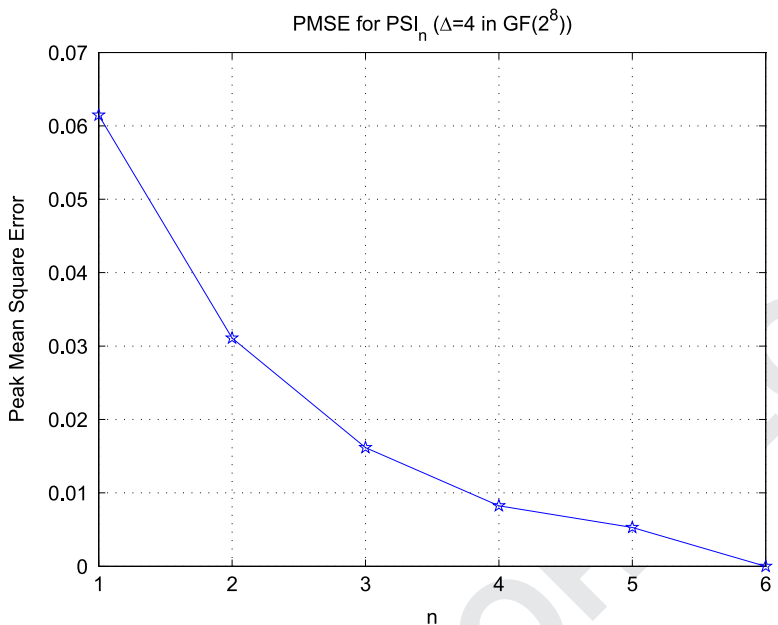

Fig. 7. Average PMSE for $\mathrm{PSI}_{n}$. $\mathrm{PSI}_{n}(1 \leq n \leq 6)$ denote cases where $\left\{\Delta_{1}, \ldots, \Delta_{n}\right\}$ is contained in PSI.

\section{Simulation results}

In this section, we quantitatively evaluate the performance of the proposed approaches and compare them with other existing approaches such as the approach presented in [14], Strategy 1 (S1) discussed in Section 3.2 (denoted by the SI scheme), and the method described by (17) (denoted by the PSI scheme). We then deploy the proposed approach in a sensor network scenario.

\subsection{Performance comparison of approximate decoding algorithms}

Unlike the approaches shown in [14] and the SI scheme, the proposed PSI scheme requires additional information as a form of PSI, which can be considered as an overhead. The overhead is defined in the simulations as the ratio of the amount of data additionally added to the amount of

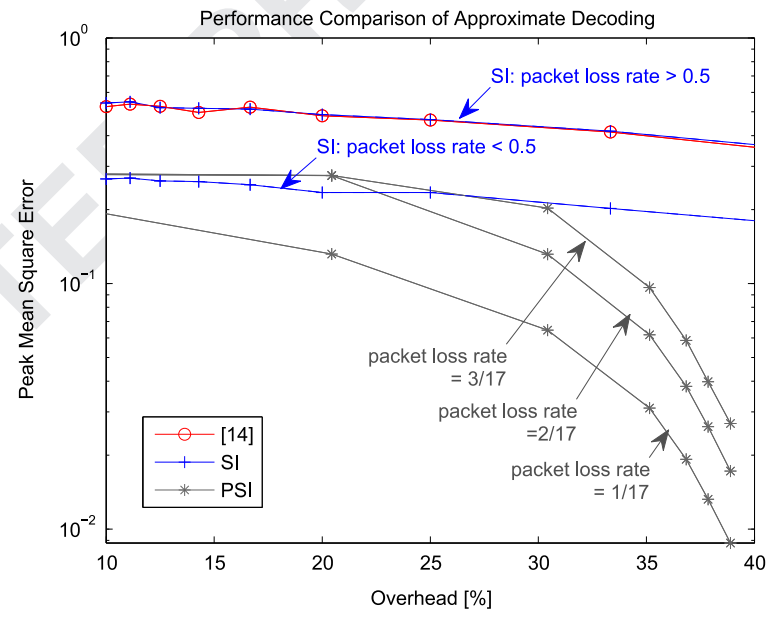

Fig. 8. Performance comparison for three different approximate decoding algorithms.

original data. For fair comparison, the source node in [14] and the SI scheme repeatedly transmit packets such that they have the same amount of overhead as the PSI scheme, since [14] and the SI scheme do not require additional information for decoding. For example, 25\% overhead for [14] and the SI scheme means that one packet is transmitted in addition to every four packets, resulting in five transmissions in total. In case of the PSI scheme, however, the amount of additional information included in PSI is $25 \%$ larger than the total packet size (i.e., four packets).

The simulation results for performance comparison are shown in Fig. 8. In the simulations, $\Delta=8$ and 17 packets are network coded over $G F\left(2^{10}\right)$. Fig. 8 shows the average PMSE for the considered approaches over 100 independent experiments. For the SI scheme, the packet loss rates are set in the range of $(0,0.5]$ and $(0.5,1)$, and the corresponding average PMSEs shown in Fig. 8 are indicated by "SI: packet loss rate $<0.5$ " and "SI: packet loss rate $>0.5$ ",
85

101

105

107

109

111 
respectively. For the PSI scheme, the packet loss rates are set to $1 / 17,2 / 17$ and $3 / 17$ for illustration.

It can be easily observed that the performance of all the approaches improves as the overhead increases. This is because the additional information can help the decoder to recover more data correctly. In particular, the impact of the additional information on the performance improvement of the PSI scheme is the greatest among the considered approaches, which is consistent with the analytical results discussed in Section 3.2. It is also observed that the performance curves converge into two PMSE levels as the overhead approaches $0 \%$, which are determined based on the packet loss rates, i.e., packet loss rate is higher or lower than $50 \%$. If the packet loss rate is higher than $50 \%$, approximate decoding needs more artificially generated information (e.g., using (4)) than innovative (received) packets, thereby leading to performance degradation.

\subsection{Illustrative application: approximate decoding deploy- ment in sensor networks}

In this section, we consider an illustrative application of the proposed approximate decoding algorithm with PSI in seismographic networks. In this simulation, we use seismic data that is actually collected from 30 sensors that measure the amplitude of a seismic signal at a distance of $100 \mathrm{~m}$ from each other. A sensor $t$ captures signal $\mathbf{s}_{t}$ that represents a series of sampled observations in a time window of size $L$, i.e., $\mathbf{s}_{t}=\left[s_{t}^{(1)}, \ldots, s_{t}^{(L)}\right]^{T}$. The measured symbol is quantized and mapped into $G F\left(2^{M}\right)$ elements, and the resulting data is denoted by $x_{t}$, i.e., $\mathcal{Q}\left(s_{t}^{(i)}\right)=x_{t}^{(i)} \in$ $G F\left(2^{M}\right)$. Each sensor makes measurements and forwards them to its neighbor encoding sensors. An index vector of sensors whose data are encoded together at sensor $t$ is denoted by $\mathbf{H}_{i} \in t$, where $i$ is a vector index. An encoding sensor $t$ may receive multiple data from sensors in $\mathbf{H}_{i}$ and combine them based on RLNC, where the combined data is again forwarded to its neighbor nodes or the final destination (i.e., receiver). The elements of coding coefficient vector $\mathbf{c}_{t}$ are randomly selected from $G F\left(2^{M}\right)$. The encoded data packets generated at the first coding stage are expressed as $\mathbf{y}^{(i)}(2)=\sum_{t \in \mathbf{H}_{i}} \oplus \mathbf{c}_{t}(1) \otimes x_{t}^{(i)}$. The encoded packets are mixed again at the intermediate nodes while traversing network towards the destination. An illustrative example for simulation setup is shown in Fig. 9.

As a representative example, we consider the case where the data measured from sensors 1-3 are collected and combined by sensor 3 based on RLNC in our experiments. The measured signals from a set of sensors are mostly time-shifted and energy-scaled. The energy difference between sensors is modeled as the Gaussian distribution. The similarity of the signals becomes higher as sensors are closer to each other. In this simulation, the energy difference between the signals captured by neighbor sensors is modeled as Gaussian random variable with mean value of 8 and variance of $0.2^{2}$, i.e., $s_{t+1}^{(i)}-s_{t}^{(i)} \sim \mathcal{N}\left(8,0.2^{2}\right)$ for $t=1,2$. In Fig. 10, the seismic data measured from three sensors are depicted. We set the temporal window size by $L=256$ for data representation and use the quantizer $\mathbf{x}_{t} \in G F\left(2^{M}\right)$ for source data quantization. The $G F$ size is set to $2^{7}$, i.e., $G F\left(2^{7}\right)$ and packet loss rate is set to $1 / 3$. The receiver reconstructs the original source data based on the data collected from the three sensors.

In the decoding process, we deploy SI as $\nu$, i.e., $\boldsymbol{\nu}=8 \cdot \mathbf{1}_{\left(T-K_{\text {in }}\right)}$, in (4). This is the S1 discussed in Section 3.2. We also deploy the proposed decoding algorithm including the PSI, which is discussed in Section 4. The results are captured by sensor 3 and Fig. 11(a-2) shows the decoded signal based on S1 that depends only on SI. Fig. 11(a-3) and (a-4) shows the decoded signals based on PSI with $\Delta_{2}$ and with $\Delta_{2}$ and $\Delta_{3}$, respectively. The average PMSE is presented in Table 5, where the amount of decoding error is shown in Fig. 11. Fig. 11(a-1) shows the original signal

63

Fig. 9. An illustrative example of sensor network for seismic data. Sensors are deployed and capture seismic signals. The symbols $s_{1}^{(i)}, s_{2}^{(i)}$, and $s_{3}^{(i)}$ measured at sensors 1,2 and 3, respectively, are transformed into GF (i.e., $x_{1}^{(i)}, x_{2}^{(i)}, x_{3}^{(i)}$ ) and encoded based on RLNC. In this example, $y_{k}^{(i)}(2)=\left\{c_{k 1}(1) \otimes x_{1}^{(i)}\right\} \oplus\left\{c_{k 2}(1) \otimes x_{2}^{(i)}\right\} \oplus\left\{c_{k 3}(1) \otimes x_{3}^{(i)}\right\}$. The encoded data forwarded to their neighbor nodes through error-prone networks. Some packets can be lost in the transmission and the receiver reconstructs source data based on the proposed approximate decoding algorithm. 
reduced to $17.3 \%$ and $6.7 \%$ of (a-2), in (a-3) and (a-4), respectively. As discussed, the performance of approximate decoding improves as more $\Delta_{i}$ is included in PSI.

\section{Related works}

In this section, we study prior work related with the proposed approaches. Several regularization techniques [28] can be deployed for overcoming the all-or-nothing problem of network coding in finite fields. For example, the pseudo-inverse of underdetermined coefficient matrix
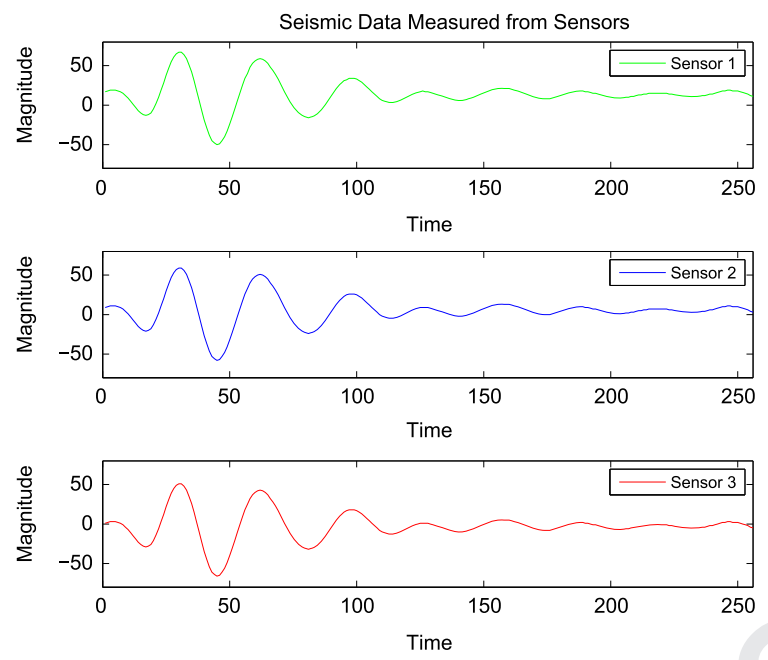

Fig. 10. Seismic data measured from sensors. Sensor 1 measures the seismic data and forwards it to sensor 2 with energy attenuation with $\mathcal{N}\left(8,0.2^{2}\right)$. can be used for decoding. However, it is generally known that this type of regularization techniques may result in unreasonable approximations [28]. Alternatively, Tikhonov regularization [29] can improve the decoding performance by slightly modifying the standard least square approach. However, this technique cannot be easily deployed in practice because it requires to determine additional optimization parameters. Sparsity assumptions can also be used for regularized decoding in underdetermined systems in cases where a model of the signal of interest is known a priori [30]. However, all of these regularization techniques have been designed and developed in the field of real numbers, but not in finite fields that are used in network coding approaches. Hence, they may show significant performance degradation if they are blindly deployed in the proposed framework, as they cannot consider several properties (e.g., cyclic properties) of finite field operations. Underdetermined systems can also be solved approximately based on the maximum likelihood estimation (MLE) techniques (see e.g., Part II of [31]) or based on mixed integer linear programming [32]. However, these techniques require effective data models and may typically involve large computational complexity.

Unlike the approaches mentioned above, several approaches have been proposed to overcome the all-ornothing problem of network coding in finite fields (see e.g., [33,32]). In [33], a loss-tolerant protocol for broadcasting, named by Dragoncast, based on network coding is proposed. In [32], a coding scheme that jointly considers both network coding and multiple description coding is proposed. The problem of data reconstruction is formulated as a mixed integer quadratic programming and robustness against missing packets is achieved. Our prior work [14] propose an approximate decoding approach based on

(a-1) Seismic Signal Measured from Sensors

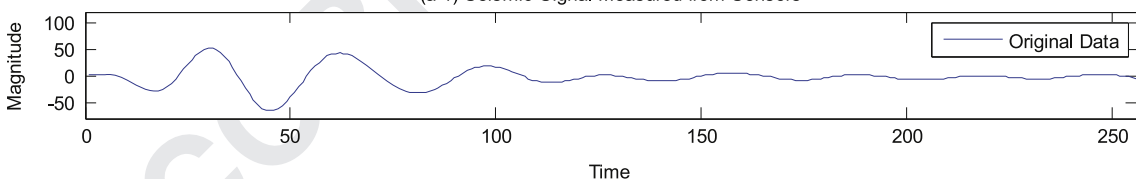

(a-2) Decoded Signal with $\mathrm{SI}(\Delta)$

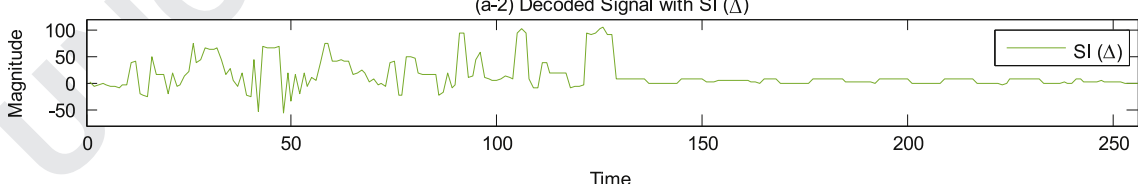

(a-3) Decoded Signal with $\mathrm{PSI}\left(\Delta, \Delta_{2}\right)$

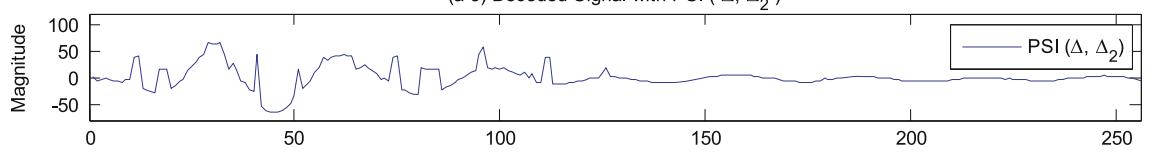

(a-4) Decoded Signal with PSI $\left(\Delta, \Delta_{2}, \Delta_{3}\right)$

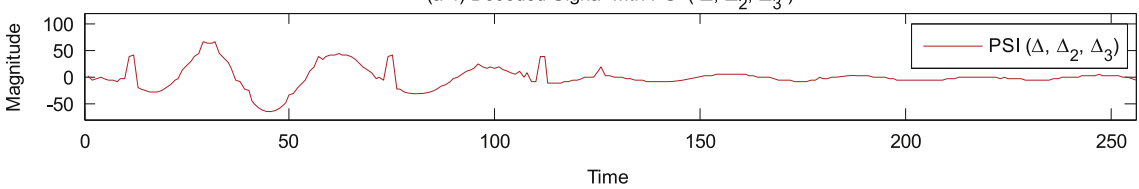

Fig. 11. Seismic signals measured from sensors and decoded signals by approximate decoding with PSI. Energy difference on the signals is modeled by Gaussian distribution, $\mathcal{N}\left(8,0.2^{2}\right)$, and network coding and approximate decoding are performed in $G F\left(2^{7}\right)$. 
Table 5

Average PMSE for different approximate decoding algorithms.

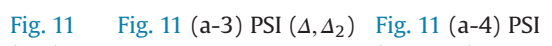

PMSE(\%) $0.0010 \quad 1.7266 \times 10^{-4}(17.3 \%) \quad 6.7118 \times 10^{-5}(6.7 \%)$ $(100 \%)$

9

11 simple matches of the most similar data between neighbor sources.

The information on the inter-dependency of the sources has been exploited in order to improve the performance of data reconstruction. In [34], network coding for two arbitrary inter-dependent sources is studied in a generic network and an upper bound on the probability of decoding errors is found as a function of the network parameters such as the number of links upstream of a receiver or network topologies. Several practical aspects of joint source and network coding are investigated in [35]. However, the proposed approach is sub-optimal and provides a solution only for two inter-dependent sources that are transmitted over binary symmetric channels. The design of optimal network codes under joint distributed source and network coding framework is studied in a sensor network [36]. The goal is to find the optimal tradeoff between compression efficiency and network robustness. However, the complexity of the proposed solution in [36] grows exponentially with the network size. In [23], an iterative decoding algorithm for source reconstruction is proposed for inter-dependent sources following a belief propagation approach that incorporates inter-dependence characteristics. However, none of prior works solve the all-or-nothing problem of network coding in finite fields with low-complexity, while the algorithm proposed in this paper can be implemented with lowcomplexity and it is compatible with well-known Gaussian elimination methods. Several extensions of [14] can be found in [15] and [16] for deterministic sources with SI and PSI, respectively. In [17], approximate decoding algorithm for sources that have bell-shaped, symmetric and unimodal distribution is proposed. Unlike these prior works, we propose more generalized framework that can cover a large range of source types. Moreover, the proposed approximate decoding solution can exploit both SI and PSI such that the approximate decoding performance can be significantly improved.

Since additional information is transmitted [37] in the proposed scenario, the proposed approach can be considered as an index coding problem [38]. However, the index coding is generally considered in broadcasting scenarios (i.e., a single server wishes to communicate with several clients) and the side information in the index coding contains the source information [39]. Hence, the amount of additional information required for the proposed approach is significantly smaller than that for index coding.

\section{Conclusion}

In this paper, we consider the transmission of network coded inter-dependent sources in error prone networks. In order to solve the all-or-nothing problem of network coding approaches, we propose a solution that approximately reconstructs the source data when the number of received data packets is not sufficient for perfect recovery without major change in the conventional decoding architecture. Unlike prior works, we consider a generalized source inter-dependency characterized by unimodal shapes. Given the information about source distributions, we show that the performance of the proposed approximate decoding algorithm is improved by using the mode of the distributions (SI). We further improve the performance of the proposed approximate decoding algorithms by deploying additional information called PSI. We evaluate the proposed approaches in an illustrative example of sensor network and the simulation results confirm that approximate decoding along with minimal side information leads to effective source reconstruction.

\section{Appendix A}

In Appendix A, we show that the approximate decoding performance is maximized only when $\nu_{n}=\nu_{n}^{*}$ and is flat for all other value of $\nu_{n}\left(\neq \nu_{n}^{*}\right)$ values, which justify the use of the Cauchy-Dirac delta function in Section 3.2.

The approximate decoding algorithm in (4) can be expressed as

$$
\begin{aligned}
& \hat{\mathbf{x}}^{(i)}=\left[\begin{array}{c}
\mathbf{C}\left(h_{D}-1\right) \\
\mathbf{D}
\end{array}\right]-1\left[\begin{array}{c}
\mathbf{y}^{(i)}\left(h_{D}\right) \\
\nu
\end{array}\right]=\overline{\mathbf{C}} \\
& \odot\left[\begin{array}{c}
\mathbf{y}^{(i)}\left(h_{D}\right) \\
\nu
\end{array}\right]=\left[\overline{\mathbf{c}}_{1}, \ldots, \overline{\mathbf{c}}_{K_{i n}}, \overline{\mathbf{c}}_{K_{\text {in }}+1}, \ldots, \overline{\mathbf{c}}_{T}\right] \\
& \odot\left[\begin{array}{c}
\mathbf{y}^{(i)}\left(h_{D}\right) \\
\nu
\end{array}\right]=\left[\overline{\mathbf{c}}_{1}, \ldots, \overline{\mathbf{c}}_{K_{\text {in }}}\right] \odot \mathbf{y}^{(i)}\left(h_{D}\right) \oplus\left[\overline{\mathbf{c}}_{K_{\text {in }}+1}, \ldots, \overline{\mathbf{c}}_{T}\right] \\
& \odot \nu
\end{aligned}
$$

where $\overline{\mathbf{C}} \triangleq\left[\mathbf{C}\left(h_{D}-1\right)^{T}, \mathbf{D}^{T}\right]^{-T}=\left[\overline{\mathbf{c}}_{1}, \ldots, \overline{\mathbf{c}}_{T}\right]$ and $\overline{\mathbf{c}}_{t}$ is in $G F\left(2^{M}\right)$. Hence, the squared decoding error can be expressed as

$\left|\mathbf{x}^{(i)}-\hat{\mathbf{x}}^{(i)}\right|^{2}=\left|\left[\overline{\mathbf{c}}_{K_{i n}+1}, \ldots, \overline{\mathbf{c}}_{T}\right] \odot\left(\nu^{* T}-\nu^{T}\right)\right|^{2}$

The squared decoding error shown in (21) implies that $\nu=\nu^{*}$ is the condition that minimizes the error. However, a small distance between $\nu$ and $\nu^{*}\left(\nu \neq \nu^{*}\right)$ in the GF does not directly lead to small distance of $\left|\mathbf{x}^{(i)}-\hat{\mathbf{x}}^{(i)}\right|^{2}$ due to the cyclic property of the operations in the GF and the random selection of the coding coefficients. The characteristics of the decoding performance (i.e., the squared decoding error) can thus be well captured by the Cauchy-Dirac delta function. An illustrative example shown in Fig. 12 for the squared decoding error in (21) also confirms the discussion above. 


\section{5}

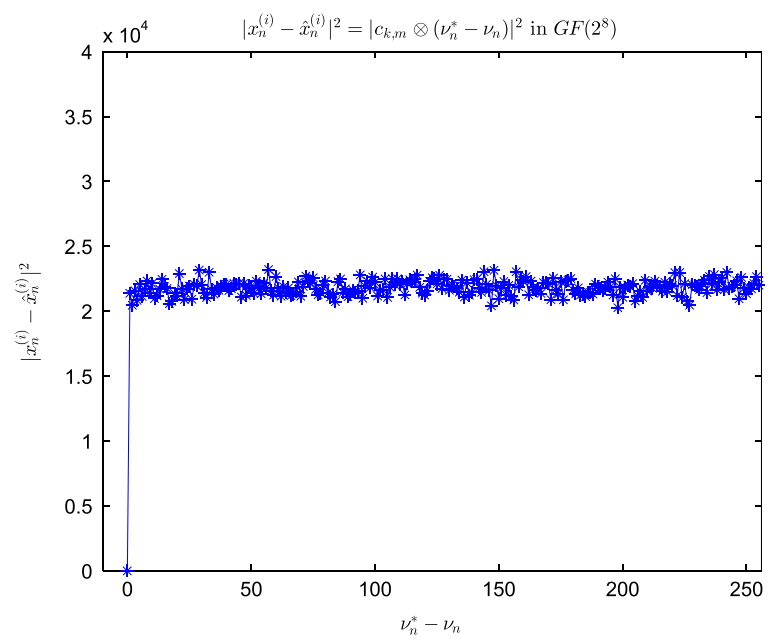

Fig. 12. An illustrative example of the squared decoding error $\left|x_{n}^{(i)}-\hat{x}_{n}^{(i)}\right|^{2}=\left|c_{k, m} \otimes\left(\nu_{n}^{*}-\nu_{n}\right)\right|^{2}$ in $\operatorname{GF}\left(2^{8}\right) . \quad c_{k, m}$ is randomly selected in $\mathrm{GF}\left(2^{8}\right)$.

\section{References}

[1] R. Ahlswede, N. Cai, S.-Y.R. Li, R.W. Yeung, Network information flow, IEEE Trans. Inf. Theory 46 (July (4)) (2000) 1204-1216.

[2] S.-Y.R. Li, R.W. Yeung, N. Cai, Linear network coding, IEEE Trans. Inf. Theory 49 (February (2)) (2003) 371-381.

[3] Z. Li, B. Li, D. Jiang, L.C. Lau, On achieving optimal throughput with network coding, in: IEEE International Conference on Computer and Communications (INFOCOM), vol. 3, Miami, FL, USA, March 2005, pp. 2184-2194.

[4] P.A. Chou, Y. Wu, Network coding for the internet and wireless networks, IEEE Signal Process. Mag. 24 (September (5)) (2007) 77-85.

[5] T. Ho, R. Koetter, M.Médard, D. Karger, M. Effros, The benefits of coding over routing in a randomized setting, in: IEEE International Symposium on Information Theory, Cambridge, MA, USA, June-July 2003.

[6] C. Gkantsidis, P.R. Rodriguez, Network coding for large scale content distribution, in: IEEE International Conference on Computer and Communications (INFOCOM), vol. 4, March 2005, pp. 2235-2245.

[7] S. Acedański, S. Deb, M. Médard, R. Koetter, How good is random linear coding based distributed networked storage, in: Workshop on Network Coding, Theory and Applications, Riva del Garda, Italy, April 2005.

[8] S. Deb, M. Médard, C. Choute, On random network coding based information dissemination, in: IEEE International Symposium on Information Theory, Adelaide, Australia, September 2005, pp. 278282.

[9] C. Gkantsidis, J. Miller, P. Rodriguez, Comprehensive view of a live network coding P2P system, in: ACM SIGCOMM Conference on Internet Measurement, Brazil, October 2006.

[10] A.G. Dimakis, P.B. Godfrey, M.J. Wainwright, K. Ramchandran, Network coding for distributed storage systems, in: IEEE International Conference on Computer and Communications (INFOCOM), Anchorage, AK, USA, May 2007.

[11] T. Ho, M. Médard, J. Shi, M. Effros, D.R. Karger, On randomized network coding, in: Annual Allerton Conference on Communication, Control, and Computing, Monticello, IL, USA, October 2003.

[12] M. Médard, R. Koetter, D. Karger, M. Effros, J. Shi, B. Leong, A random linear network coding approach to multicast, IEEE Trans. Inf. Theory 52 (October (10)) (2006) 4413-4430.

[13] C. Fragouli, E. Soljanin, Network coding applications, Found. Trends Netw. 2 (2007).

[14] H. Park, N. Thomos, P. Frossard, Approximate decoding approaches for network coded correlated data, Signal Process. 93 (January (1)) (2013) 109-213.
[15] M. Kwon, H. Park, An improved approximate decoding with correlated sources, in: SPIE Optical Engineering + Applications. International Society for Optics and Photonics, San Diego, CA, USA, August 2011.

[16] M. Kwon, H. Park, P. Frossard, Improved approximate decoding based on position information matrix, in: IEEE Symposium on Computers and Communications, Cappadocia, Turkey, July 2012.

[17] M. Kwon, H. Park, Approximate recovery of network coded real-time information, in: International Conference on Information Networking (ICOIN), Phuket, Thailand, February 2014, pp. 545-549.

[18] K.-J. Bathe, E.L. Wilson, Numerical Methods in Finite Element Analysis, Prentice-Hall, Englewood Cliffs, NJ, 1976

[19] P. Paweczak, S. Pollin, H.W. So, A. Bahai, R. Prasad, R. Hekmat, Comparison of opportunistic spectrum multichannel medium access control protocols, in: IEEE Global Telecommunications Conference (GLOBECOM2008), 2008, pp. 1-6.

[20] B.F. Lo, A survey of common control channel design in cognitive radio networks, Phys. Commun. 4 (1) (2011) 26-39.

[21] K. Bian, J. Park, R. Chen, Control channel establishment in cognitive radio networks using channel hopping, IEEE J. Sel. Areas Commun. 29 (4) (2011) 689-703.

[22] L. Lazos, S. Liu, M. Krunz, Mitigating control-channel jamming attacks in multi-channel ad hoc networks, in: Proceedings of the Second ACM Conference on Wireless Network Security, 2009, pp. 169-180.

[23] E. Bourtsoulatze, N. Thomos, P. Frossard, Correlation-aware reconstruction of network coded sources, in: IEEE International Symposium on Network Coding, Cambridge, MA, USA, June 2012.

[24] J. Liu, Q. Yang, B. Yao, R. Brown, G. Yue, Linear correlation between fractal dimension of eeg signal and handgrip force, Biol. Cybern. 93 (2) (2005) 131-140.

[25] R. Bacastow, C. Keeling, T. Whorf, Seasonal amplitude increase in atmospheric $\mathrm{CO}_{2}$ concentration at Mauna Loa, Hawaii, 1959-1982, J. Geophys. Res.: Atmos. (1984-2012) 90 (D6) (1985) 10529-10540.

[26] A.M. Eskicioglu, P.S. Fisher, Image quality measures and their performance, IEEE Trans. Commun. 43 (12) (1995) 2959-2965.

[27] M.G. Katz, D. Tall, A Cauchy-Dirac delta function, Found. Sci. 18 (1) (2013) 107-123.

[28] A. Neumaier, Solving ill-conditioned and singular linear systems: a tutorial on regularization, SIAM Rev. 40 (September (3)) (1998) 636-666.

[29] G.H. Golub, P.C. Hansen, D.P. O'Leary, Tikhonov regularization and total least squares, SIAM J. Matrix Anal. Appl. 21 (1) (1999) 185-194.

[30] E.J. Candès, M.B. Wakin, An introduction to compressive sampling, IEEE Signal Process. Mag. 25 (March (2)) (2008) 21-30.

[31] S. Bhattacharyya, Handbook of Signal Processing Systems, Springer, 2010.

[32] L. Iwaza, M. Kieffer, L. Liberti, K. Al-Agha, Joint decoding of multipledescription network-coded data, in: International Symposium on Network Coding, Beijing, China, July 2011.

[33] S.Y. Cho, C. Adjih, Wireless broadcast with network coding in mobile ad-hoc networks: Dragoncast, in: Proceedings of the EU-Korea Conference on Science and Technology, vol. 124, 2008, pp. 333-344.

[34] T. Ho, M. Médard, M. Effros, R. Koetter, Network coding for correlated sources, in: Conference on Information Sciences and Systems, Princeton, NJ, USA, March 2004.

[35] Y. Wu, V. Stankovic, Z. Xiong, S. Y. Kung, On practical design for joint distributed source and network coding, in Workshop on Network Coding, Theory, and Applications, Riva del Garda, Italy, April 2005.

[36] X. Zhang, S.B. Wicker, Robustness vs. efficiency in sensor network, in: International Symposium on Information Processing in Sensor Networks, Los Angeles, CA, USA, April 2005.

[37] Z. Bar-Yossef, Y. Birk, T.S. Jayram, T. Kol, Index coding with side information, IEEE Trans. Inf. Theory 57 (March 3) (2011) 1479-1494.

[38] Y. Birk, T. Kol, Informed-source coding-on-demand (iscod) over broadcast channels, in: Seventeenth Annual Joint Conference of the IEEE Computer and Communications Societies (INFOCOM 1998), vol. 3, March 1998, pp. 1257-1264.

[39] M. Effros, S. El Rouayheb, M. Langberg, An equivalence between network coding and index coding, in: IEEE International Symposium on Information Theory Proceedings (ISIT 2013), July 2013, pp. 967-971.
61 